\title{
ON UNIQUENESS OF SOLUTIONS OF $n$-TH ORDER DIFFERENTIAL EQUATIONS IN CONFORMAL GEOMETRY
}

\author{
Sun-Yung A. Chang and Paul C. Yang
}

\section{$\S 1$. Introduction and statement of theorem}

In this paper, we prove a uniqueness theorem for an $n$-th order elliptic equation on the standard $n$-sphere $S^{n}$. The problem arises naturally from the point of view of conformal geometry. The method we use is the method of moving planes originated in Alexandrov [A] and Gidas-Ni-Nirenberg [G-N-N].

On the unit sphere $S^{2}$ with standard metric $g_{0}$, when one makes a conformal change of the metric $g=e^{2 w} g_{0}$, the Gaussian curvature $K=K(g)$ satisfies the differential equation

$$
\Delta w+K e^{2 w}=1
$$

on $S^{2}$, where $\Delta$ denotes the Laplacian operator with respect to the metric $g_{0}$ on $S^{2}$.

When $K \equiv 1$ on (1.1), as a result of the Cartan-Hadamard theorem, we have $e^{2 w} g_{0}$ is the pull back of the standard metric through some conformal transformation $\varphi$ (i.e. $g$ is isometric to $g_{0}$ ) or equivalently $w=\frac{1}{2} \log \left|J_{\varphi}\right|$, where $J_{\varphi}$ denotes the Jacobian of the transformation $\varphi$.

In [C-L], Chen and Li studied the corresponding equation of (1.1) on $\mathbb{R}^{2}$ with $K \equiv 1$, and they proved, using the method of moving plane, the stronger result that when $u$ is a smooth function defined on $\mathbb{R}^{2}$ satisfying

$$
-\Delta u=e^{2 u} \quad \text { on } \quad \mathbb{R}^{2}
$$

with $\int_{\mathbb{R}^{2}} e^{2 u} d x<\infty$, then $u(x)$ is symmetric with respect to some point $x_{0} \in \mathbb{R}^{2}$ and there exists some $\lambda>0$, so that $u(x)=\log \frac{2 \lambda}{\lambda^{2}+\left|x-x_{0}\right|^{2}}$ on $\mathbb{R}^{2}$. There is an alternative argument of Chanillo-Kiessling ([C-K]) for this uniqueness result via a strict isoperimetric inequality.

When $n \geq 3$, a natural generalization of the Gaussian-curvature equation (1.1) above is the scalar curvature equation under conformal change of metric. On $\left(S^{n}, g_{0}\right)$, denote $g=u^{\frac{4}{n-2}} g_{0}$ the conformal change of metric of $g_{0}$, where

Received September 29, 1996.

Research of first author is supported in part by NSF Grant DMS-9401465.

Research of second author is supported in part by NSF Grant DMS-9300881. 
$u$ is a positive function, then the scalar curvature $R=R(g)$ of the metric is determined by the following differential equation

$$
c_{n} \Delta u+R u^{\frac{n+2}{n-2}}=R_{0} u,
$$

where $c_{n}=\frac{4(n-1)}{n-2}, R_{0}=n(n-1)$. When $R=R_{0}$, a uniqueness result established by Obata [O] again states that this happens if the metric $g$ is isometric to $g_{0}$ or equivalently $u=\left|J_{\varphi}\right|^{\frac{n-2}{2 n}}$ for some conformal transformation $\varphi$ of $S^{n}$. In [C-G-S] Caffarelli-Gidas-Spruck studied the corresponding equation of (1.3) on $\mathbb{R}^{n}$ :

$$
-\Delta u=n(n-2) u^{\frac{n+2}{n-2}}, \quad u>0 \quad \text { on } \quad \mathbb{R}^{n}
$$

They classified all solutions of (1.4), via the method of moving plane, as $u(x)=$ $\frac{2 \lambda}{\lambda^{2}+\left|x-x_{0}\right|^{2}}$ for some $x_{0} \in \mathbb{R}^{n}, \lambda>0$.

In this paper, we study another set of equations which are also natural generalization of the equation (1.1). To state our result, we first recall the notion of conformal covariant operators.

On a general compact manifold $M$ with metric $g$, a metrically defined operator $A$ is said to be conformally covariant if under the conformal change in metric $g_{w}=e^{2 w} g$, the pair of corresponding operators $A_{w}$ and $A$ are related by

$$
A_{w}(\varphi)=e^{-b w} A\left(e^{a w} \varphi\right)
$$

for all $\varphi \in C^{\infty}(M)$. Basic examples of such operators are: when $n=2, A=$ the Laplace operator $\Delta$, then $a=0 ; b=2$; when $n \geq 3, L=-c_{n} \Delta+R$, then $a=\frac{n-2}{2}, b=\frac{n+2}{2}$. It turns out on compact 4-manifold, an interesting 4 th-order operator was discovered by Paneitz $[\mathrm{P}]$ :

$$
P_{4} \varphi=\Delta^{2} \varphi+\delta\left(\frac{2}{3} \operatorname{Rg}-2 \operatorname{Ric}\right) d \varphi
$$

where $\delta$ denotes the divergence, $d$ the differential, and Ric the Ricci tensor of the metric $g$. Under the conformal change $g_{w}=e^{2 w} g, P_{4}$ undergoes the transformation $\left(P_{4}\right)_{w}=e^{-4 w} P_{4}$ (i.e. $\left.a=0, b=4\right)$. On general compact manifold of dimension $n$, the existence of such an operator $P_{n}$ with $\left(P_{n}\right)_{w}=$ $e^{-n w} P_{n}$ for even dimensional manifold is verified in [G-J-M-S]. However, it is only explicitly known for the Euclidean space $\mathbb{R}^{n}$ with standard metric $\left(P_{n}=\right.$ $\left.(-\Delta)^{n / 2}\right)$ and hence for the sphere $S^{n}$ with standard metric $g_{0}$. The explicit formula for $P_{n}$ on $S_{n}$ has appeared in Branson [B-1] and Beckner [Be] as follows:

$$
\left\{\begin{array}{l}
\text { For } n \text { even } P_{n}=\prod_{k=0}^{\frac{n-2}{2}}(-\Delta+k(n-k-1)), \\
\text { For } n \text { odd } P_{n}=\left(-\Delta+\left(\frac{n-1}{2}\right)^{2}\right)^{1 / 2} \prod_{k=0}^{\frac{n-3}{2}}(-\Delta+k(n-k-1)) .
\end{array}\right.
$$


On general compact manifolds, it turns out in the cases when the dimension of the manifold is 3 or 4 , there exist some natural curvature invariant $Q_{n}$ of order $n$ which, under conformal change of metric $g_{w}=e^{2 w} g$, is related to $P_{n} w$ satisfying the following differential equation:

$$
-P_{n} w+\left(Q_{n}\right)_{w} e^{n w}=\left(Q_{n}\right)_{0} \quad \text { on } \quad M .
$$

The reader is referred to articles $[\mathrm{C}-\mathrm{Y}]$ for a study of the equation (1.6) in the case $n=4$, to [C-Q-1] and [C-Q-2] for the existence of an operator $P_{3}$ and a curvature invariant $Q_{3}$ satisfying equation (1.6) defined on boundaries of 4manifolds. And to [B-1] and the survey article $[\mathrm{C}]$ for a discussion of general properties of Paneitz operators.

On $\left(S^{n}, g_{0}\right)$, when the metric $g_{w}$ is isometric to the standard metric, then $\left(Q_{n}\right)_{w}=\left(Q_{n}\right)_{0}=(n-1)$ ! . In this case, equation (1.6) becomes

$$
-P_{n} w+(n-1) ! e^{n w}=(n-1) ! \quad \text { on } S^{n} .
$$

In this paper, we will establish the following uniqueness result for solutions of equation (1.7).

Theorem 1.1. On $\left(S^{n}, g_{0}\right)$, all smooth solution of the equation (1.7) are of the form $e^{2 w} g_{0}=\varphi^{*}\left(g_{0}\right)$ for some conformal transformation $\varphi$ of $S^{n}$; i.e. $w=$ $\frac{1}{n} \log \left|J_{\varphi}\right|$ for the transformation $\varphi$.

We reformulate the equation (1.7) on $\mathbb{R}^{n}$. For each point $\xi \in S^{n}$, denote $x$ its corresponding point under the sterographic projection $\pi$ from $S^{n}$ to $\mathbb{R}^{n}$, sending the north pole on $S^{n}$ to $\infty$; i.e. Suppose $\xi=\left(\xi_{1}, \xi_{2}, \ldots, \xi_{n+1}\right)$ is a point $\in S^{n} \subset$ $\mathbb{R}^{n+1}, x=\left(x_{1}, \ldots, x_{n}\right) \in \mathbb{R}^{n}$, then $\xi_{i}=\frac{2 x_{i}}{\left.|+| x\right|^{2}}$ for $1 \leq i \leq n ; \xi_{n+1}=\frac{1-|x|^{2}}{1+|x|^{2}}$. Suppose $w$ is a smooth function on $S^{n}$, denote $\varphi(x)=\log \frac{2}{1+|x|^{2}}=\log \left|J_{\pi^{-1}}\right|$, $u(x)=\varphi(x)+w(\xi)$. Since the Paneitz operator $P_{n}$ is the pull back under $\pi$ of the operator $(-\Delta)^{n / 2}$ on $\mathbb{R}^{n}$ ([c.f. B-2, Theorem 3.3$]$ ), $w$ satisfies the equation (1.6) on $S^{n}$ if and only if $u$ satisfies the corresponding equation

$$
(-\Delta)^{n / 2} u=(n-1) ! e^{n u} \quad \text { on } \quad \mathbb{R}^{n} .
$$

Thus Theorem 1.1 above is equivalent to the following result:

Theorem 1.2. On $\mathbb{R}^{n}$, suppose $u$ is a smooth function satisfies the equation (1.8). Suppose in addition that

$$
u(x)=\log \frac{2}{1+|x|^{2}}+w(\xi(x)),
$$

for some smooth function $w$ defined on $S^{n}$, then $u(x)$ is symmetric w.r.t. some point $x_{0} \in \mathbb{R}^{n}$, and there exists some $\lambda>0$ so that

$$
u(x)=\log \frac{2 \lambda}{\lambda^{2}+\left|x-x_{0}\right|^{2}} \quad \text { for all } \quad x \in \mathbb{R}^{n} .
$$


We remark that, in the case when $w$ is a minimal solution of the functional with Euler-Lagrange equation (1.7), the result in Theorem 1.1 is a consequence of some sharp Sobolev type inequalities of Milin-Lebedev when $n=1$, Moser $[\mathrm{M}]$ and Onofri [On] when $n=2$, and Beckner [Be] for general $n$. The reader is also referred to $[\mathrm{C}]$ for a general discussion of the relation between sharp inequalities and equations of type (1.7).

We would like also to acknowledge that during the course of preparation of the paper, the above theorem was independently proved by C. S. Lin [L] and $\mathrm{X}$. $\mathrm{Xu}[\mathrm{Xu}]$ when $n=4$ for functions satisfying equation (1.8) under some less restrictive growth conditions at infinity which are analytic in nature. In general, it remains open whether there exist some natural geometric conditions under which functions satisfying equation (1.8) are necessarily of the form (1.9).

We organize the paper as follows. In section 2 , we describe briefly the method of moving planes and some main ideas of our proof. In Section 3, we establish some technical lemmas; mainly to establish a form of Hopf's Lemma for the pseudo-differential operator $(-\Delta)^{1 / 2}$ and to extend some technical facts in [CG-S] to the operator $(-\Delta)^{1 / 2}$. In Section 4 , we prove Theorem 1.2.

\section{$\S 2$. Method of moving plane}

We describe briefly the method of moving plane. First we recall a fundamental result of Gidas-Ni-Nirenberg established by using the method.

Theorem 2.1 [G-N-N]. Suppose $u$ is a positive $\mathcal{C}^{2}$ function satisfying

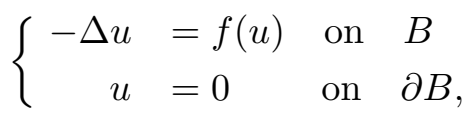

in the unit ball $B$ in $\mathbb{R}^{n}$ and $f$ is a Lipschitz function. Then $u(x)=u(|x|)$ is a radial symmetric decreasing function in $r=|x|$ for all $x \in B$.

To set up the proof in $[\mathrm{G}-\mathrm{N}-\mathrm{N}]$ of above theorem we introduce the following notations. For each point $x \in \mathbb{R}^{n}$, denote $x=\left(x_{1}, x^{\prime}\right)$ where $x_{1} \in \mathbb{R}, x^{\prime} \in \mathbb{R}^{n-1}$. For each real number $\lambda$, denote

$$
\begin{aligned}
\Sigma_{\lambda} & =\left\{x=\left(x_{1}, x^{\prime}\right) \mid x_{1}<\lambda\right\}, \\
T_{\lambda} & =\left\{x=\left(x_{1}, x^{\prime}\right) \mid x_{1}=\lambda\right\}, \\
x_{\lambda} & =\left(2 \lambda-x_{1}, x^{\prime}\right) \quad \text { the reflection point of } x \quad \text { w.r.t. } T_{\lambda} .
\end{aligned}
$$

Define

$$
w_{\lambda}(x)=u(x)-u\left(x_{\lambda}\right) \equiv u(x)-u_{\lambda}(x) .
$$

Suppose $u$ satisfies equation (2.1), the idea of moving plane is to prove that $w_{\lambda}(x) \geq 0$ on $\Sigma_{\lambda}$ for all $0 \leq \lambda \leq 1$ and actually $w_{\lambda=0} \equiv 0$. This is achieved via maximum principle and Hopf's boundary lemma to the function $u$. Thus $u\left(x_{1}, x^{\prime}\right)=u\left(-x_{1}, x^{\prime}\right)$ for $x \in B$. Since one can repeat this argument for any 
hyper-plane passing through the origin of the ball $B$, one establishes that $u$ is radially symmetric w.r.t. to the origin.

If one attempts to generalize above argument to higher order elliptic equation such as $(-\Delta)^{2} u=f(u)$ with suitable boundary conditions, one quickly realizes that, due to a lack of maximum principle for higher order elliptic equation, such result in general cannot be expected to hold. Nevertheless, it turns out that for a special class of Lipschitz functions $f$; namely for functions $f$ satisfying $f(0) \geq 0$ with $f$ monotonically increasing, e.g. $f(u)=e^{u}$, one can modify the argument in $[\mathrm{G}-\mathrm{N}-\mathrm{N}]$. The key observation is that for each $f$, if $(-\Delta)^{2} u=f(u)$ then

$$
(-\Delta)^{2} w_{\lambda}(x)=f(u)-f\left(u_{\lambda}\right)=c(x) w_{\lambda}(x)
$$

where $c(x)$ is some positive function whose value at $x$ lies between $f(u(x))$ and $f\left(u_{\lambda}(x)\right)$. From (2.2) one then concludes that $w_{\lambda}(x) \geq 0$ on $\Sigma_{\lambda}$ if and only if $(-\Delta)^{2} w_{\lambda} \geq 0$ on $\sum_{\lambda}$. Since $w_{\lambda}(x)=(-\Delta) w_{\lambda}(x)=0$ for $x \in T_{\lambda}, w_{\lambda}(x) \geq 0$ on $\sum_{\lambda}$ also happens if and if $(-\Delta) w_{\lambda}(x) \geq 0$ on $\Sigma_{\lambda}$. This suggests that one should apply the maximum principle and Hopf's lemma to the function $(-\Delta) w_{\lambda}$ to generalize result in $[\mathrm{G}-\mathrm{N}-\mathrm{N}]$ to higher order elliptic operators like $(-\Delta)^{2}$.

In Section 4 below, we prove Theorem 1.2 by applying this argument to the function $(-\Delta)^{m-1} w_{\lambda}(x)$ where $m=\left[\frac{n+1}{2}\right]$. In the case when $n$ is even $(n=2 m)$, one can then apply directly some technical lemmas (Lemma 3.3 and Lemma 3.4 quoted below in our paper) in [C-G-S] to the method of moving planes to finish the proof of Theorem 1.2. In the case when $n$ is odd $(n=2 m-1)$, we establish a form of Hopf's lemma (Lemma 3.7 below ) for the pseudo-differential operators $(-\Delta)^{1 / 2}$, and also modify the proof of Lemma 3.4 to a version adapted to the operator $(-\Delta)^{1 / 2}$, then finish the proof of Theorem 1.2.

\section{$\S 3$. Preliminary lemmas}

Suppose $u(x)=\varphi(x)+w(\xi(x))$, where $\varphi(x)=\log \frac{2}{1+|x|^{2}}$ and $w(\xi)$ is a smooth function defined on $S^{n}$, as in the statement of Theorem 1.2. We write $m=\left[\frac{n+1}{2}\right]$, i.e. $n=2 m$ when $n$ is even, $n=2 m-1$ when $\mathrm{n}$ is odd; and let $v(x)=$ $(-\Delta)^{m-1} u(x)$. The following give some preliminary estimates for $v(x)$ near $\infty$ :

\section{Lemma 3.1.}

$$
\begin{aligned}
\lim _{|x| \rightarrow \infty} u(x) & =-\infty, \\
\lim _{|x| \rightarrow \infty} e^{u(x)} & =O\left(\frac{1}{|x|^{2 n}}\right), \\
\left|\nabla^{(k)} \varphi(x)\right| & =O\left(\frac{1}{|x|^{k}}\right)=\left|\nabla^{(k)} u(x)\right| \quad \text { as }|x| \rightarrow \infty, \\
\left|\nabla^{(k)} w(x)\right| & =O\left(\frac{1}{|x|^{k+1}}\right) \quad \text { as }|x| \rightarrow \infty .
\end{aligned}
$$


Proof. All statements follow from direct computation. (d) follows inductively from the chain rule and the relation $\frac{\partial \xi_{i}}{\partial x_{j}}=\frac{2 \delta_{i j}}{1+|x|^{2}}-\frac{4 x_{i} x_{j}}{\left(1+|x|^{2}\right)^{2}}$.

We then derive the following "harmonic asymptotic expansion" at $\infty$ as in [C-G-S].

Lemma 3.2. $v$ has a harmonic asymptotic expansion at $\infty$ :

$$
\begin{aligned}
v(x) & =\frac{a_{0}}{|x|^{2(m-1)}}+\sum_{i} \frac{a_{i} x_{i}}{|x|^{2 m}}+O\left(\frac{1}{|x|^{2 m}}\right), a_{0}>0, \\
v_{x_{i}} & =-2(m-1) a_{0} \frac{x_{i}}{|x|^{2 m}}+O\left(\frac{1}{|x|^{2 m}}\right), \\
v_{x_{i} x_{j}} & =O\left(\frac{1}{|x|^{2 m}}\right) .
\end{aligned}
$$

Proof. Breaking up $(-\Delta)^{m-1} u(x)=(-\Delta)^{m-1} \varphi(x)+(-\Delta)^{m-1} w(\xi(x))$. We easily verify by direct computation that $(-\Delta)^{m-1} \varphi(x)$ has a harmonic asymptotic expansion at $\infty$ of the form above with $a_{0}>0$ and $a_{i}=0$ for $i=1, \ldots, n$. To check that $(\Delta)^{m-1} w(\xi(x))$ does not contribute to the leading order terms, we use the inversion coordinates $y_{i}=\frac{x_{i}}{|x|^{2}}$ as smooth coordinates for a neighborhood of $\infty$. We have

$$
\partial_{x_{i}} w=\sum_{\alpha} \partial_{y_{\alpha}}\left(w(\xi(y))\left(\frac{\delta_{\alpha i}}{|x|^{2}}-\frac{2 x_{\alpha} x_{i}}{|x|^{4}}\right)\right.
$$

and

$\Delta_{x} w(x)=\sum_{\alpha, \beta} \partial_{y_{\alpha}} \partial_{y_{\beta}} w(\xi(y))\left(\frac{\delta_{\alpha i}}{|x|^{2}}-\frac{2 x_{\alpha} x_{i}}{|x|^{4}}\right)\left(\frac{\delta_{\beta i}}{|x|^{2}}-\frac{2 x_{\beta} x_{i}}{|x|^{4}}\right)+\nabla_{y} w \cdot \frac{(-2 n+4) x}{|x|^{4}}$

Hence, iterating the operator $\Delta_{x} m-1$ times, we see that $(\Delta)^{m-1} w(\xi(x))$ will contribute at most a term of the form $\frac{a_{i} x_{i}}{|x|^{2 m}}$ which comes from applying $(-\Delta)^{m-2}$ to the second factor in the second term above. The remaining terms are all of order at most $\frac{1}{|x|^{2 m}}$.

The following two lemmas are Lemma 2.3 and special case of Lemma 2.4 in [C-G-S], where $\sum_{\lambda}, x_{\lambda}$ are defined as in Section 2.

Lemma 3.3. Let $v$ be a function in a neighborhood of infinity satisfying the asymptotic expansion (3.1). Then there exists large positive constants $\bar{\lambda}, \bar{R}$ such that if $\lambda \geq \bar{\lambda}$,

$$
v(x)>v\left(x_{\lambda}\right) \quad \text { for } \quad x \in \Sigma_{\lambda}, \quad|x|>\bar{R} .
$$

We remark that although above lemma was proved for the case when $2(m-$ 1) $=n-2$ with $n \geq 3$ (i.e. when $n$ is even in our case) in [C-G-S], the same proof also works for any $m \geq 2$. Actually the same proof also works when $n=2$ 
with $m=1$, or $n=1$ (with $m=1$ ) also with the leading term in the asymptotic behavior in (3.1) replaced by $\log \frac{1}{|x|}$.

A second remark is that for the case when $n$ is even, $v=(-\Delta)^{m-1} u$ is a superharmonic function on $\mathbb{R}^{n}$ satisfying $-\Delta v=e^{u}$; with $\lim _{|x| \rightarrow \infty} v(x)=0$; hence $v$ is positive.

Lemma 3.4. Let $v$ be a positive solution of $-\Delta v=F(x)$ where $v$ has a harmonic asymptotic expansion (3.1) at $\infty$. Suppose that for $x \in \Sigma_{\lambda_{0}}$ (i.e. $x_{1}<\lambda_{0}$ ) we have

$$
\begin{aligned}
v(x) & \geq v\left(x_{\lambda_{0}}\right) \quad \forall x \in \Sigma_{\lambda_{0}}, \\
v(x) & \neq v\left(x_{\lambda_{0}}\right), \\
F(x) & \geq F\left(x_{\lambda_{0}}\right) \quad \forall x \in \Sigma_{\lambda_{0}} .
\end{aligned}
$$

Then there exists some $\varepsilon>0, R_{1}>0$ such that

(i) $v_{x_{1}}<0$ on $\left|x_{1}-\lambda_{0}\right|<\varepsilon,|x|>R_{1}$,

(ii) $v\left(x_{1}, x^{\prime}\right)>v\left(2 \lambda-x_{1}, x^{\prime}\right)$ for all $x \in \Sigma_{\lambda}$ for all $\lambda$ with $\lambda-\lambda_{0}$ suitably small multiple of $\varepsilon$ with $\lambda_{0}-x_{1}>\frac{1}{2} \varepsilon>\lambda_{0}-\lambda$.

We will now state the version of classical Hopf's boundary lemma for later reference.

Lemma 3.5. Suppose $h$ satifies $\Delta h+c h \leq 0$ and $h \geq 0$ in a domain $\Omega$ of $\mathbb{R}^{n}$ with smooth boundary. Then

(a) if $h$ vanishes at some point in $\Omega$, then $h \equiv 0$ in $\Omega$,

(b) if $h \not \equiv 0$ in $\Omega$, then the exterior normal derivative $\frac{\partial h}{\partial n}<0$ on the boundary of $\Omega$.

We begin to deal with the case when $n=2 m-1$ is odd. In this case, $(-\Delta)^{n / 2}=(-\Delta)^{1 / 2} \circ(-\Delta)^{m-1}$. We first remark that the pseudo-differential operator $(-\Delta)^{1 / 2}$ can be identified as the Dirichlet-Neumann operator on $L^{2}\left(\mathbb{R}^{n}\right)$. That is, for each $h \in L^{2}\left(\mathbb{R}^{n}\right)$, denote $\tilde{h}$ its harmonic extension to the upper half-space $\mathbb{R}_{+}^{n+1}=\left\{(x, t) \mid x \in \mathbb{R}^{n}, t>0\right\}$, i.e.

$$
\tilde{h}(x, t)=\int_{\mathbb{R}^{n}} P_{t}(y) h(x-y) d y,
$$

where $P_{t}(y)=\int_{\mathbb{R}^{n}} e^{-2 \pi i x \cdot y} e^{-2 \pi|x| t} d x$ denotes the Poisson kernel of $\mathbb{R}_{+}^{n+1}$. One sees easily that $\left((-\Delta)^{1 / 2} h\right)^{\wedge}(x)=(2 \pi|x|) \hat{h}(0)=\left(-\frac{\partial}{\partial t} \tilde{h}\right)^{\wedge}(x, 0)$ where $\wedge$ denotes the Fourier transform on $\mathbb{R}^{n}$. Thus $(-\Delta)^{1 / 2}=\frac{\partial}{\partial n}$, the Dirichlet-Neumann operator.

Lemma 3.6. On $\mathbb{R}^{n},(n \geq 3)$, suppose $h, f$ are smooth functions with $h \in$ $L^{2}\left(\mathbb{R}^{n}\right), f \in L^{1}\left(\mathbb{R}^{n}\right)$ with $f(x)=O\left(\frac{1}{|x|^{\alpha}}\right)$, for some $\alpha>n / 2$ as $|x| \rightarrow \infty$ satisfying $(-\Delta)^{1 / 2} h=f$ on $\mathbb{R}^{n}$ then

$$
h(x)=\left((-\Delta)^{-1 / 2} f\right)(x)=c_{n} \int_{\mathbb{R}^{n}} \frac{1}{|x-y|^{n-1}} f(y) d y,
$$


for all $x \in \mathbb{R}^{n}$ where $c_{n}=2 \frac{\Gamma((n-1) / 2)}{\pi^{n / 2}}$.

Proof. Call $k(x)$ the expression on the right hand side of the formula (3.2), then under our assumptions on $f$, one can easily check that $k \in L^{2}\left(\mathbb{R}^{n}\right)$. Formula (3.2) then follows from the fact that $\left(\frac{1}{|x|^{n-1}}\right)^{\wedge}=\frac{1}{c_{n}}|x|^{-1}$ in the sense of distribution (c.f. for example Stein [S], P. 117), by taking Fourier transform on both side of (3.2).

The following is a version of Hopf's Lemma for the operator $(-\Delta)^{1 / 2}$ which we shall use later in our proof.

Lemma 3.7. Suppose $(-\Delta)^{1 / 2} h=f$ with $h, f$ satisfying the same assumption as in Lemma 3.5. Also that for some $\lambda \in \mathbb{R}, f(x)=-f\left(x_{\lambda}\right)$ for all $x \in \mathbb{R}^{n}$ and $f \geq 0$ on $\Sigma_{\lambda}$. Then $h \geq 0$ on $\Sigma_{\lambda},\left.\frac{\partial h}{\partial x_{1}}\right|_{T_{\lambda}} \leq 0$ and

$$
h(x)=\frac{c_{n}}{4} \int_{y \in \Sigma_{\lambda}}\left(\frac{1}{|x-y|^{n-1}}-\frac{1}{\left|x-y_{\lambda}\right|^{n-1}}\right) f(y) d y .
$$

Furthermore strict inequalities hold $h>0$ on $\Sigma_{\lambda}$ and $\frac{\partial h}{\partial x_{1}}>0$ on $T_{\lambda}$ unless $h \equiv 0$.

Proof. Let $G(x, y)=\frac{c_{n}}{|x-y|^{n-1}}$ denote the fundamental solution for $(-\Delta)^{1 / 2}$. Then $G(x, y) \geq G\left(x, y_{\lambda}\right)$ for $x, y \in \Sigma_{\lambda}$. It follows from our assumption that $f(y)=-f\left(y_{\lambda}\right)$ for all $y \in \mathbb{R}^{n}$, and the integral formula (3.2) that

$$
\begin{aligned}
h(x) & =\frac{1}{2} \int_{\mathbb{R}^{n}}\left(G(x, y)-G\left(x, y_{\lambda}\right)\right) f(y) d y \\
& =\frac{1}{4} \int_{y \in \Sigma_{\lambda}}\left(G(x, y)-G\left(x, y_{\lambda}\right)\right) f(y) d y .
\end{aligned}
$$

From this formula it follows that if $f(y) \geq 0$ for all $y \in \Sigma_{\lambda}$ then $h \geq 0$ for all $x \in \Sigma_{\lambda}$; also $h>0$ unless $f \equiv 0$. And

$$
\begin{aligned}
\left.\frac{\partial}{\partial x_{1}} h(x)\right|_{x \in T_{\lambda}} & =\frac{1}{2} \int_{y \in \Sigma_{\lambda}}\left(\left.\frac{\partial}{\partial x_{1}} G(x, y)\right|_{x \in T_{\lambda}}\right) f(y) d y \\
& =-\frac{c_{n}(n-1)}{2} \int_{y \in \Sigma_{\lambda}} \frac{\left(\lambda-y_{1}\right)}{|x-y|^{n+1}} f(y) d y<0 .
\end{aligned}
$$

We remark in the case $n=1$, statements in Lemma 3.6 and Lemma 3.7 also hold for the fundamental solution $G(x, y)=\frac{1}{2 \pi} \ln \frac{1}{|x-y|}$ This can be verified directly using Fourier transform as in the proof above, or as in the proof of Lemma 4.8 in $[\mathrm{C}-\mathrm{Y}]$.

Lemma 3.8. Suppose $(-\Delta)^{1 / 2} v=F(x)$ where $F \in L^{1}\left(\mathbb{R}^{n}\right)$ and $F(x)=O\left(\frac{1}{|x|^{\alpha}}\right)$, for some $\alpha>n / 2, n \geq 3$. Suppose $v$ and $F$ satisfying the same hypothesis $(a)$ 
and (b) as in the statement of Lemma 3.4, then the same conclusions as in Lemma 3.4 hold.

Proof. This is a modification of the proof of Lemma 2.4 in [C-G-S] adopted to our setting. Let $h(x)=v(x)-v\left(x_{\lambda_{0}}\right)$ for $x \in \Sigma_{\lambda_{0}}$. Then by the estimates in Lemma 2.1, $v, h \in L^{2}\left(\mathbb{R}^{n}\right)$. Thus we may apply Lemma 3.7 to the function $h$ and $f(x)=F(x)-F\left(x_{\lambda_{0}}\right)$ and conclude that

$$
\begin{aligned}
h(x) & =\frac{c_{n}}{4} \int_{y \in \Sigma_{\lambda_{0}}}\left(\frac{1}{|x-y|^{n-1}}-\frac{1}{\left|x-y_{\lambda_{0}}\right|^{n-1}}\right) f(y) d y \\
& =\frac{c_{n}}{4} \int_{y \in \Sigma_{\lambda_{0}}}\left(\frac{1}{|x-y|^{n-1}}-\frac{1}{\left|x_{\lambda_{0}}-y\right|^{n-1}}\right) f(y) d y .
\end{aligned}
$$

We claim that there exists some constant $c>0$ so small that

$$
h(x)>\frac{c\left(\lambda_{0}-x_{1}\right)}{|x|^{n+1}} \quad \text { for } \quad x \in \Sigma_{\lambda_{0}} \quad \text { and } \quad|x| \text { sufficiently large. }
$$

(3.5) is an easy consequence of the integral formula (3.4) as follows: For $x$, $y \in \Sigma_{\lambda_{0}}$, we have

$$
\begin{aligned}
\frac{1}{|x-y|^{n-1}}-\frac{1}{\left|x_{\lambda_{0}}-y\right|^{n-1}} & \geq c_{1} \frac{\left|x_{\lambda_{0}}-y\right|^{2}-|x-y|^{2}}{\left|x_{\lambda_{0}}-y\right|^{n+1}} \\
& =c_{1} \frac{4\left(\lambda_{0}-x_{1}\right)\left(\lambda_{0}-y_{1}\right)}{\left|x_{\lambda_{0}}-y\right|^{n+1}},
\end{aligned}
$$

for some dimensional constant $c_{1}$. Hence for $x \in \Sigma_{\lambda_{0}}$

$$
\begin{aligned}
h(x) & \geq c_{2} \int_{y \in \Sigma_{\lambda_{0}}} \frac{\left(\lambda_{0}-x_{1}\right)\left(\lambda_{0}-y_{1}\right)}{\left|x_{\lambda_{0}}-y\right|^{n+1}} f(y) d y \\
& \geq c_{2}\left(\lambda_{0}-x_{1}\right) \int_{\left|x_{\lambda_{0}}-y\right| \leq 2|x|} \lambda_{0}-y_{1} \geq 1 \\
& \geq c \frac{f(y)}{\left|x_{\lambda_{0}}-y\right|^{n+1}} d y \\
|x|^{n+1} &
\end{aligned}
$$

for $x \in \Sigma_{\lambda_{0}},|x|$ large where $c_{2}$ is again a dimensional constant and $c$ is a constant close to $c_{2} \int_{\lambda_{0}-y_{1} \geq 1} f(y) d y$ when $|x|$ is sufficiently large. Thus (3.5) holds.

Once (3.5) is established, we may apply the same argument as in the proof of Lemma 2.4 in [C-G-S] to establish Lemma 3.7.

We again remark that statement of Lemma 3.8 holds for $n=1$ also.

\section{$\S 4$. Proof of Theorem 1.2}

We will show that there exists some $\lambda_{0}$ so that $w_{\lambda_{0}} \equiv 0$. Since the same argument applies after any rotation of the coordinate system, we conclude that 
there exists some point $x_{0} \in \mathbb{R}^{n}$ with respect to which $u$ is radial symmetric. The result then follows from the uniqueness of the O.D.E. solution $u=u\left(\left|x-x_{0}\right|\right)=$ $u(r)$ for equation (1.8).

For a given function $u$, let $v=(-\Delta)^{m-1} u$ be defined as before. We will establish $w_{\lambda_{0}} \equiv 0$ in two steps.

Step 1. There exists some positive $\lambda_{1}$, if $\lambda \geq \lambda_{1}$, then $w_{\lambda}(x) \geq 0$ for all $x \in \Sigma_{\lambda}$.

Choose $\bar{R}$, and $\bar{\lambda}$ as in the statement of Lemma 3.3 , then for $\lambda \geq \bar{\lambda}, x \in \Sigma_{\lambda}$ and $|x| \geq \bar{R}$, we have $v(x)>v\left(x_{\lambda}\right)$. Since $v$ is a positive function according to elliptic theory for the case $n$ is even or via formula (3.2) in Lemma 3.6 when $n$ is odd and $n \geq 3$, and in either cases we have $\lim _{|x| \rightarrow \infty} v(x)=0$, we may choose $\lambda_{1}$ sufficiently large so that if $\lambda \geq \lambda_{1}$, then $v(x)>v\left(x_{\lambda}\right)$ for all $x \in \Sigma_{\lambda}$. In the special case when $n=1$, then $v \equiv u$, we can modify above argument using the expression that $u(x)=\log \frac{2}{1+|x|^{2}}+\omega(\xi(x))$ for some bounded function $\omega$ to draw the same conclusion for $v$.

Once we have $v(x)>v\left(x_{\lambda}\right)$ for $x \in \Sigma_{\lambda}$, since $(-\Delta)^{k}\left(w_{\lambda}\right)(x)=0$ for all $x \in T_{\lambda}$ the boundary of $\Sigma_{\lambda}$, for all $0 \leq k \leq m-1$, Step 1 follows by applying the maximal principle of the Laplacian iteratively.

Step 2 . Let $\lambda_{0}$ be the smallest value $\lambda$ so that $w_{\lambda} \geq 0$ on $\Sigma_{\lambda}$, for each $\lambda \geq \lambda_{0}$. We claim:

(i) For $\lambda>\lambda_{0}$, we have $w_{\lambda}(x)>0$ for $x \in T_{\lambda}$ and $\frac{\partial}{\partial x_{1}} v(x)<0$ for $x \in \Sigma_{\lambda}$.

(ii) $w_{\lambda_{0}} \equiv 0$ for $x \in \Sigma_{\lambda_{0}}$.

To establish (i), we notice that for $\lambda \geq \lambda_{0}$

(a) $w_{\lambda}(x) \geq 0$ for $x \in \Sigma_{\lambda}$ for all $\lambda \geq \lambda_{0}$ hence $\frac{\partial}{\partial x_{1}} u(x) \leq 0$ for $x \in T_{\lambda_{0}}$,

(b) $w_{\lambda}(x) \geq 0$ on $\Sigma_{\lambda}$, hence $(-\Delta)^{n / 2} w_{\lambda} \geq 0$ on $\Sigma_{\lambda}$ by equation (1.8). Denote $h_{\lambda}(x)=v(x)-v\left(x_{\lambda}\right)$. Then in the case $n=2 m$ we have $h_{\lambda}=0$ on $T_{\lambda}$ and $(-\Delta) h_{\lambda} \geq 0$ on $\Sigma_{\lambda}$ hence $h_{\lambda} \geq 0$ on $\Sigma_{\lambda}$ which in turn implies that $\frac{\partial}{\partial x_{1}} v(x) \leq 0$ for all $x \in T_{\lambda}$. In the case $n=2 m-1$ is odd, we can draw the same conclusion by applying Lemma 3.7 to the function $h=h_{\lambda}$ and $f=e^{n u(x)}-e^{n u\left(x_{\lambda}\right)}$.

(c) Since $h_{\lambda}=(-\Delta)^{m-1} w_{\lambda}(x) \geq 0$ on $\Sigma_{\lambda}$, in the cases $m \geq 2$ we may apply maximal principle to $w_{\lambda}(x)$ and conclude that either $w_{\lambda}(x)>0$ or $w_{\lambda}(x) \equiv 0$ on $\Sigma_{\lambda}$, and the later happens if and only if $\frac{\partial}{\partial x_{1}} w_{\lambda}=2 \frac{\partial u}{\partial x_{1}}=0$ for some point $x \in T_{\lambda}$.

(d) Applying similar argument as in (b), using Lemma 3.5 and Lemma 3.7 respectively for $n$ even and odd cases, we conclude that either $h_{\lambda}>0$ or $h_{\lambda}(x) \equiv$ 0 on $\Sigma_{\lambda}$ and the later happens if and only if $\frac{\partial v}{\partial x_{1}}(x)=\frac{1}{2} \frac{\partial}{\partial x_{1}} h_{\lambda}(x)=0$ for some point $x \in T_{\lambda}$.

We now prove claim (i) in Step 2.

We already know that for $\lambda \geq \lambda_{0}, w_{\lambda}(x) \geq 0$ on $\Sigma_{\lambda}$. Suppose for some $\lambda>\lambda_{0}$ say $\lambda=\lambda_{0}+\delta$ we have $w_{\lambda_{0}+\delta}(x)=0$ for some $x \in \Sigma_{\lambda}$, hence $w_{\lambda_{0}+\delta}(x) \equiv 0$. It follows that $u\left(\lambda_{0}, x^{\prime}\right)=u\left(\lambda_{0}+2 \delta, x^{\prime}\right)$ and hence $\frac{\partial u}{\partial x_{1}}\left(\lambda_{0}+2 \delta, x^{\prime}\right)=0$, and hence $w_{\lambda_{0}+2 \delta} \equiv 0$. From this, inductively we may conclude $u(x)=u(x+2 k \delta)$ for all $k \geq 1$ hence $u(x)=u(\infty)=-\infty$ for all $x \in \Sigma_{\lambda}$ which is a clear contradiction. Thus $w_{\lambda}(x)>0$ for all $x \in \Sigma_{\lambda}, \lambda>\lambda_{0}$. 
From this point on, we may apply the equation (1.8) and repeat the same argument as in the previous paragraph using instead facts in (b), (d) to the function $h_{\lambda}=v(x)-v\left(x_{\lambda}\right)$ to conclude that $h_{\lambda}(x)>0$ for all $x \in \Sigma_{\lambda}$. We can then apply either Lemma 3.5 or 3.7 to establish claim (i).

To prove claim (ii), if we assume the contrary, then by our argument above we have

$$
h_{\lambda_{0}}(x)>0 \quad \text { on } \quad \Sigma_{\lambda_{0}} \quad \text { and } \quad \frac{\partial}{\partial x_{1}} h_{\lambda_{0}}<0 \quad \text { on } \quad T_{\lambda_{0}} .
$$

On the other hand by our definition of $\lambda_{0}$, there exists a increasing sequence of $\lambda_{k}, \lambda_{k} \rightarrow \lambda_{0}$ and $x_{k} \in \Sigma_{\lambda_{k}}$ so that $w_{\lambda_{k}}\left(x_{k}\right)<0$. Applying equation (1.8), we conclude that there exists points $y_{k} \in \Sigma_{\lambda_{k}}$ with $h_{\lambda_{k}}\left(y_{k}\right)<0$ and in particular $h_{\lambda_{k}}\left(z_{k}\right)=\min _{y \in \Sigma \lambda_{k}} h_{\lambda_{k}}(y)<0$. We choose $\bar{R}$ and $\varepsilon$ as in the statement of Lemma 3.4 and Lemma 3.8 respectively for the case $n$ is even or $n$ odd, to conclude that when $k$ is sufficient large so that when $\lambda_{0}-\lambda_{k}<\frac{1}{4} \varepsilon$, then we have $\left|z_{k}\right|<\bar{R}$ for $k$ large. Thus some subsequence of $z_{k}$ converges to some point $z_{0} \in \Sigma_{\lambda_{0}} \cup T_{\lambda_{0}}$, which satisfies

$$
h_{\lambda_{0}}\left(z_{0}\right) \leq 0 \quad \text { and } \quad \nabla h_{\lambda_{0}}\left(z_{0}\right)=0 \text {. }
$$

This contradicts (4.1), and we thus established claim (ii) and finished the proof of Theorem 1.2.

\section{Acknowledgements}

The authors greatly benefited from many discussions with Lihe Wang about the method of moving planes. We would like also to thank L. Caffarelli for some helpful suggestion which lead us to prove the theorem for the case when $n$ is odd.

\section{References}

[A] A. D. Alexandrov, Uniqueness theorems for surfaces in the large, Vestnik Leningrad. Univ. Mat. Mekh. Astronom. 13 (1958), 5-8; Amer. Math. Soc. Transl. Ser. 221 (1962), 412-416.

[Be] W. Beckner, Sharp Sobolev inequalities on the sphere and the Moser-Trudinger inequality, Ann. of Math. 138 (1993), 213-242.

[B-1] T. Branson, Group representations arising from Lorentz conformal geometry, J. Funct. Anal. 74 (1987), 199-293.

[B-2] Sharp inequality, the functional determinant and the complementary series, Trans. Amer. Math. Soc. 347 (1995), 3671-3742.

[C-G-L] L. Caffarelli, B. Gidas, and J. Spruck, Asymptotic symmetry and local behavior of semi-liner equations with critical Sobolev growth, Comm. Pure Appl. Math. 42 (1989), 271-289.

[C] S-Y. A. Chang, On a fourth order differential operator -the Paneitz operator-in conformal geometry, preprint, Proceedings Conference for the 70th birthday of A. P. Calderon (to appear). 
[C-K] S. Chanillo and M. K.-H. Kiessling, Conformally invariant systems of non-linear PDEs of Liouville type, preprint.

[C-Q-1] S-Y. A. Chang and J. Qing, The functional determinants on 4-manifolds with boundary I - the formula, preprint, J. Funct. Anal. (1995) (to appear).

[C-Q-2] ㄴ The functional determinants on 4-manifolds with boundary II, preprint, J. Funct. Anal. (1995) (to appear).

[C-Y] S-Y. A. Chang and P. C. Yang, Extremal metrics of zeta functional determinants on 4-Manifolds, Ann. of Math. 142 (1995), 171-212.

[C-L] W. Chen and C. Li, Classification of solutions of some non-linear elliptic equations, Duke Math. J. 63 (1991), 615-622.

[G-N-N] B. Gidas, N. W. Ni, and L. Nirenberg, Symmetry and related properties via the maximum principle, Comm. Math. Phys. 68 (1979), 209-243.

[G-J-M-L] C. R. Graham, R. Jenne, L. Mason, and G. Sparling, Conformally invariant powers of the Laplacian, I: existence, J. London Math. Soc. (2) 46 (1992), 557-565.

[L] C.-S. Lin, A classification of solutions of a conformally invariant fourth order equation in $R^{n}$, preprint (1996).

[M] J. Moser, A sharp form of an inequality by N. Trudinger, Indiana Univ. Math. J. 20 (1971), 1077-1091.

[Ob] M. Obata, Certain conditions for a Riemannian manifold to be isometric with a sphere, Jour. Math. Soc. Japan 14 (1962), 333-340.

[On] E. Onofri, On the positivity of the effective action in a theory of random surfaces, Comm. Math. Phys. 86 (1982), 321-326.

[P] S. Paneitz, A quartic conformally covariant differential operator for arbitrary pseudo-Riemannian manifolds, preprint (1983).

[St] E. Stein, Singular integrals and differentiability properties of functions, Princeton Math. Ser, 30, Princeton University Press, Princeton, 1970.

[X] X. Xu, Classification of solutions of certain fourth order nonlinear elliptic equations in $R^{4}$, preprint (1996).

Department of Mathematics, University of California, Los Angeles, CA 900951555

E-mail address: chang@math.ucla.edu

Department of Mathematics, University of Southern California, Los Angeles, CA 90089

E-mail address: pyang@math.usc.edu 